\title{
Guided Plasmon Modes of a Graphene-Coated Kerr Slab
}

\author{
${\text { Hodjat Hajian' }{ }^{1} \text { Ivan D. Rukhlenko }}^{2,3}$ - P. T. Leung ${ }^{4}$ Humeyra Caglayan ${ }^{1,5}$. \\ Ekmel Ozbay ${ }^{1,6,7}$
}

Received: 27 June 2015 / Accepted: 28 September 2015 / Published online: 14 October 2015

(C) Springer Science+Business Media New York 2015

\begin{abstract}
We study analytically propagating surface plasmon modes of a Kerr slab sandwiched between two graphene layers. We show that some of the modes that propagate forward at low field intensities start propagating with negative slope of dispersion and positive flux of energy (fast-light surface plasmons) when the field intensity becomes high. We also discover that our structure supports an additional branch of low-intensity fast-light guided modes. The possibility of dynamically switching between the forward and the fast-light plasmon modes by changing the intensity of the excitation light or the chemical potential of the graphene layers opens up wide opportunities
\end{abstract}

Ivan D. Rukhlenko

rukhlenko.ivan@gmail.com

Hodjat Hajian

hodjat.hajian@bilkent.edu.tr

1 Nanotechnology Research Center, Bilkent University, 06800 Ankara, Turkey

2 Modeling and Design of Nanostructures Laboratory, ITMO University, Saint Petersburg 197101, Russia

3 Monash University, Clayton Campus, Victoria 3800, Australia

4 Department of Physics, Portland State University, P.O. Box 751, Portland, OR 97207-0751, USA

5 Department of Electrical and Electronics Engineering, Abdullah Gul University, 38039 Kayseri, Turkey

6 Department of Physics, Bilkent University, 06800 Ankara, Turkey

7 Department of Electrical and Electronics Engineering, Bilkent University, 06800 Ankara, Turkey for controlling light with light and electrical signals on the nanoscale.

Keywords Surface plasmons · Plasmonics $\cdot$ Kerr effect . Nonlinear optics at surfaces

\section{Introduction}

Graphene is a two-dimensional periodic array of carbon atoms arranged in a honeycomb lattice [1]. It has recently received a great deal of attention due to its potential applications in optoelectronics [2] and plasmonics [3-6]. The surface conductivity of graphene $\left(\sigma_{\mathrm{g}}\right)$ can be effectively modulated via tuning its chemical potential $(\mu)$ through chemical doping, or electrostatic or magnetostatic gating [1]. For $\operatorname{Im} \sigma_{\mathrm{g}}>0$, graphene behaves as a very thin metal layer capable of supporting transverse magnetic (TM) surface plasmons (SPs) [7-13]. Tunability of plasmon resonance through the variation of $\mu$, together with a relatively large propagation length and a small localization scale of SPs in the infrared (IR) and terahertz (THz) ranges, are key advantages of graphene SPs over those supported by noble metals like silver and gold [7]. A double layer or parallelplate waveguide (PPW) of graphene also supports SPs [14], which are strongly localized between the layers and have propagation length exceeding that of a monolayer graphene [15]. Besides having remarkable sensing and guiding applications, this structure can be used in miniaturized nonlinear optical couplers [16] and modulators [17].

It has been recently reported that the enhanced near field of SPs supported by graphene monolayer facilitates strong light-matter interaction at $\mathrm{THz}$ and IR frequencies [18]. Such interaction is beneficial for nonlinear applications of graphene-enabled heterostructures, allowing one to 
make nonlinear effects pronounced even at moderate optical powers. Therefore, considering graphene as a material with a nonlinear optical response [19], nonlinear propagation of light in a monolayer [20] and a double-layer [16] of graphene have been studied theoretically. It has been also found through pure analytical and numerical studies that using a Kerr-type nonlinear medium as the substrate, cladding or core medium in a metallic waveguide considerably affects the characteristics of SPs supported by such structures [21-25]. These characteristics of a graphene sheet on a Kerr-type nonlinear substrate [26] and a graphene PPW bounded by the Kerr media [27] have been recently studied while neglecting the nonlinear optical response of graphene. This approximation is well justified provided that a nonlinear material with considerably large Kerr nonlinearity is assumed in the calculations.

Here, using the method of refs. [24, 25], we derive an exact dispersion relation for the TM SPs of a symmetric slot waveguide formed by a nonlinear Kerr slab of width $d$ and permittivity $\varepsilon_{1}=\varepsilon_{1}^{1}+\alpha|\mathbf{E}|^{2}$, which is sandwiched between two graphene monolayers at $x= \pm d / 2$ (see Fig. 1). In accordance with the existing literature, this structure is referred to as graphene/Kerr-medium/graphene (GKG) PPW. For the sake of simplicity, it is assumed to be bounded by a linear medium of permittivity $\varepsilon_{2}$. The result is then used to analyze the properties of SPs for different geometric and material parameters, including slot thickness, chemical potential, and the nonlinear part of the Kerr permittivity.

\section{Dispersion Relation for Guided Plasmons}

The schematic of the system considered in this paper is illustrated in Fig. 1.

Following ref. [27], we shall neglect the nonlinear optical response of graphene and describe its two layers using a linear surface conductivity $\sigma_{\mathrm{g}}$, which is a function of frequency $(\omega)$, temperature of the structure $(T)$, electron relaxation rate $(\tau)$, and chemical potential $(\mu)$ [28]. This conductivity is

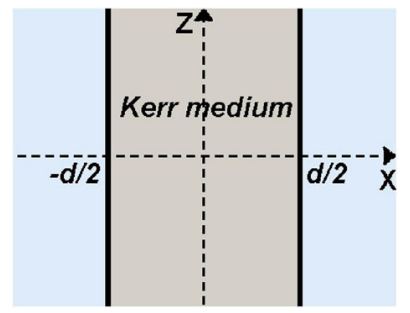

Fig. 1 Schematic of the GKG PPW. The thin black lines represented at $x= \pm d / 2$ show graphene layers inbetween the nonlinear Kerr material $\left(\varepsilon_{1}=\varepsilon_{1}^{1}+\alpha|\mathbf{E}|^{2}\right)$ is sandwiched. The structure is surrounded with a linear material of $\varepsilon_{2}=4$ generally a complex-valued function, the real part of which is responsible for the absorption losses in graphene [3, 7, $14,15]$. In order to derive an analytical dispersion relation for the guided plasmon modes of our waveguide, we shall restrict our consideration to frequencies exceeding $8 \mathrm{THz}$, for which the absorption losses in graphene can be neglected and its conductivity can be assumed purely imaginary. The electric field of the propagating modes in the $j$ th medium $(j=1,2), \mathbf{E}_{j}=\left(E_{x j} \hat{x}+i E_{z j} \hat{z}\right) e^{i \beta z}$, can then be described in terms of the $x$-dependent, real field components $E_{x j}$ and $E_{z j}$ satisfying the equations

$\frac{\mathrm{d} E_{z j}}{\mathrm{~d} x}=\frac{q_{j}^{2}}{\beta} E_{x j}$,

$\frac{\mathrm{d} E_{x j}}{\mathrm{~d} x}+\frac{E_{x j}}{\varepsilon_{j}} \frac{\mathrm{d} \varepsilon_{j}}{\mathrm{~d} x}=\beta E_{z j}$,

where $q_{j}^{2}=\beta^{2}-\varepsilon_{j} k_{0}^{2}, k_{0}=\omega / c$ is the free-space wave vector, and $\beta$ is the real propagation constant in the $z$ direction. We would like to reiterate that the absence of energy dissipation in the system $(\operatorname{Im} \beta=0)$ is a direct consequence of our simplifying assumption $\operatorname{Re} \sigma_{\mathrm{g}}=0$.

It follows from Eq. 1a that

$\left.\frac{\mathrm{d} E_{z 1}}{\mathrm{~d} x}\right|_{x=d / 2}=\frac{\left(q_{1}^{-}\right)^{2}}{\beta} E_{x 1}(d / 2)$,

where $\left(q_{1}^{-}\right)^{2}=\beta^{2}-\varepsilon_{1}^{-} k_{0}^{2}, \varepsilon_{1}^{-}=\varepsilon_{1}^{1}+\alpha E_{1}^{2}(d / 2)$, and $E_{1}^{2}(d / 2)=E_{x 1}^{2}(d / 2)+E_{z 1}^{2}(d / 2)$. By taking the $z$ field component in the surrounding medium $x \geq d / 2$ in the form

$E_{z 2}(x)=E_{z 2}(d / 2) e^{-q_{2}(x-d / 2)}$

and applying the standard boundary conditions $[15,27,28]$ for the TM wave at the graphene layer $x=d / 2$,

$E_{z 1}(d / 2)=E_{z 2}(d / 2)$,

$\left.\left(\frac{\varepsilon_{2}}{q_{2}^{2}} \frac{\mathrm{d} E_{z 2}}{\mathrm{~d} x}-\frac{\varepsilon_{1}^{-}}{\left(q_{1}^{-}\right)^{2}} \frac{\mathrm{d} E_{z 1}}{\mathrm{~d} x}\right)\right|_{x=\frac{d}{2}}=-\frac{\sigma_{\mathrm{g}}^{\prime \prime}}{\epsilon_{0} \omega} E_{z 2}(d / 2)$,

we obtain the relation

$\left.\frac{\mathrm{d} E_{z 1}}{\mathrm{~d} x}\right|_{x=d / 2}=\frac{\Delta\left(q_{1}^{-}\right)^{2}}{\varepsilon_{1}^{-}} E_{z 1}(d / 2)$,

where $\Delta=-\varepsilon_{2} / q_{2}+\sigma_{\mathrm{g}}^{\prime \prime} /\left(\omega \epsilon_{0}\right)$ and $\sigma_{\mathrm{g}}^{\prime \prime}=\operatorname{Im} \sigma_{\mathrm{g}}$.

Using Eqs. 2 and 6, one can relate the two field components in medium 1 at the waveguide interface as $E_{z 1}(d / 2)=$ $\varepsilon_{1}^{-} E_{x 1}(d / 2) /(\beta \Delta)$. This relation yields:

$\alpha E_{x 1}^{2}(d / 2)=\frac{\varepsilon_{1}^{-}-\varepsilon_{1}^{1}}{1+\left(\varepsilon_{1}^{-}\right)^{2} /(\beta \Delta)^{2}}$.

To find the exact dispersion relation for the SPs supported by the GKG PPW, we now need to know the dependency $E_{z 1}\left(E_{x 1}\right)$, which requires knowing how $\varepsilon_{1}$ varies with $E_{x 1}$ 
throughout the Kerr medium. By solving the conservation law given in Eq. 2 of ref. [24],

$\varepsilon_{1}\left[\varepsilon_{1}-2\left(2-\chi \varepsilon_{1}\right) \alpha E_{x 1}^{2}\right]=C$,

we then obtain for $x>0$

$\varepsilon_{1}\left(E_{x 1}\right)=\frac{2 \alpha E_{x 1}^{2}+X\left(E_{x 1}\right)}{1+2 \chi \alpha E_{x 1}^{2}}$,

where $X\left(E_{x 1}\right)=\sqrt{\left(2 \alpha E_{x 1}^{2}\right)^{2}+C\left(1+2 \chi \alpha E_{x 1}^{2}\right)}$ and $\chi=\left(k_{0} / \beta\right)^{2}$. The space-independent constant $C$ can be expressed through the electric field amplitude at the center of the waveguide. By denoting this amplitude as $E_{0}$ and introducing a new notation $\varepsilon_{10}=\varepsilon_{1}^{1}+\alpha E_{0}^{2}$, we obtain $C=\varepsilon_{10}\left[\varepsilon_{10}-2\left(2-\chi \varepsilon_{10}\right) \alpha E_{0}^{2}\right]$ for the symmetric mode characterized by an even function $E_{x 1}(x)$, and $C=\varepsilon_{10}^{2}$ for the antisymmetric mode characterized by an odd $E_{x 1}(x)$. Equations 7 and 8 now lead to the following fourth-order algebraic equation with respect to the unknown permittivity $\varepsilon_{1}^{-}$:

$\varepsilon_{1}^{-}-\frac{C}{\varepsilon_{1}^{-}}=\frac{2\left(2-\chi \varepsilon_{1}^{-}\right)\left(\varepsilon_{1}^{-}-\varepsilon_{1}^{1}\right)}{1+\left(\varepsilon_{1}^{-}\right)^{2} /(\beta \Delta)^{2}}$.

Solving this equation for a given $E_{0}$ allows one to find the field components at the interface $x=d / 2$, and calculate the field distribution over the entire structure.

By finally applying Eq. 1b to medium 1 and using Eq. 9, we arrive at the dispersion relation $\omega(\beta)$ in the implicit form

$\frac{\beta d}{2}=\int_{E_{x 1}(0)}^{E_{x 1}(d / 2)} \frac{\mathrm{d} E_{x 1}}{E_{z 1}\left(E_{x 1}\right)} \frac{\varepsilon_{1}\left(E_{x 1}\right)+2 \alpha E_{x 1}^{2}}{X\left(E_{x 1}\right)}$,

where the lower limit is $E_{0}$ for the symmetric mode and zero for the antisymmetric mode.

As it should be, in the absence of graphene layers $\left(\sigma_{\mathrm{g}}=\right.$ 0), Eq. 7 turns to Eq. 9 of ref. [24] and, thus, Eq. 11 reduces to the dispersion relation of the SPs supported by a simple nonlinear slot waveguide. In addition, as it has been proven for a plasmonic waveguide [24, 25], we recover the dispersion relation of a symmetric graphene-covered PPW with a linear core medium when $\alpha \rightarrow 0$.

\section{Results and Discussion}

To study the dispersion properties of GKG PPWs, we take the following material parameters: $\varepsilon_{1}^{1}=2.5, \varepsilon_{2}=4$, $T=300 \mathrm{~K}, \tau=1.35 \times 10^{-13} \mathrm{~s}$ and $\mu=0.2 \mathrm{eV}$. To be able to ignore the nonlinear response of graphene in the calculations, a highly nonlinear self-focusing material with $\alpha=6.4 \times 10^{-12} \mathrm{~m}^{2} \mathrm{~V}^{-2}$ is also chosen [21, 27]. Furthermore, considering $\sigma_{g}^{\operatorname{lin}} \mathbf{E}$ in the calculations are justified as long as the optical frequency is above $1 \mathrm{THz}$ for the modal field strength of the order of $\sim 10^{6} \mathrm{~V} / \mathrm{m}$.
Dispersions of the guided plasmonic modes of the considered GKG PPW are shown in Fig. 1 for two waveguide thicknesses, $d=10$ and $50 \mathrm{~nm}$, and two values of the nonlinear part of $\varepsilon_{1}$ at the waveguide center, $\alpha E_{0}^{2}=10^{-4}$ and 0.7 . These values correspond to the nonlinear change in the permittivity of about 0.004 and $28 \%$. One can see that our waveguide supports three branches of guided plasmon modes for $\alpha E_{0}^{2}=0.7$ and $d=10 \mathrm{~nm}$. These modes are the typical antisymmetric and symmetric branches, and an extra branch of antisymmetric SPs, corresponding to negative values of $\Delta$. Note that a linear graphene PPW can only support the ordinary forward-propagating antisymmetric and symmetric SPs, which are shown in Fig. 2a by the black solid and dashed-dotted curves [by forward-propagating we mean the modes with positive group $\left(v_{g}\right)$ and phase velocities $\left(v_{p h}\right)$ ]. Notice that supporting extra SPs by GKG PPW is directly a consequence of the interactions between the graphene SPs and the original modes of the Kerr slab.

It is also seen from Fig. $2 \mathrm{a}$ that the dispersions of typical antisymmetric and symmetric SPs roll downward for wave numbers exceeding critical values $\beta_{c 1 A} d=3.85$ and $\beta_{c 1 S} d=4.175$. This implies that highly confined modes with negative slope of dispersion can also be supported by the waveguide (check Fig. $3 \mathrm{c}$ to see how the modes are confined). Similar to the backward SPs of a nonlinear plasmonic slot waveguide [24, 25, 29], the modes with negative dispersion of the GKG PPW (we call them fast-light SPS) are associated with much stronger electric fields than the modes traveling forward for $\beta<\beta_{c 1}$. This can be seen by comparing the typical values of $E_{x}$ and $E_{z}$ in Fig. $3 \mathrm{c}$ with similar values in Fig. 3a, b. It is worth noting that, for the backward modes supported by the metal-insulator-metal waveguides, the total energy flux in the propagation direction of SPs is negative and therefore $v_{g}$ and energy velocity $\left(v_{e}\right)$ lay in the same direction, antiparallel to $v_{p h}$ [30].

To be able to investigate the physical origin of the modes with negative dispersions represented in Fig. 2a, we calculated the averaged total energy flux of the SPs in the propagation direction, $\widehat{z}\left(\frac{1}{2} \operatorname{Re}\left\{\int\left(\left[\vec{E} \times \vec{H}^{*}\right] \cdot \hat{z}\right) d x\right\}\right)$. It was found that since there is not any material with negative permittivity in the $\mathrm{x}$ direction, the total energy flux is positive for the guided modes. Therefore, the power propagates in the same direction as the phase, and thus there is not any supported backward SP by the graphene-coated Kerr slab. To make the type of the SPs with negative dispersion in our system clear, following ref. 30, we used causality and reinvestigated dispersion of the modes. By considering a source placed at $z \rightarrow-\infty$ and launching power in the positive direction $\widehat{z}$, we applied a tiny loss (from either graphene or the surrounding dielectric material) in the calculations and obtained dispersion of the modal effective index of the SPs, $n_{\text {eff }}=\beta \omega / c$ where $\beta=\beta^{\prime}+i \beta^{\prime \prime}$. The numerically calculated dispersions revealed that the trend of the dispersion 
Fig. 2 Panels a, b show the dispersions of symmetric (S) and antisymmetric (A) SP modes supported by the GKG PPW with $\alpha E_{0}^{2}=0.7$ for $d=10$ and $50 \mathrm{~nm}$, respectively. Panels c, d are the same as (a) and (b) with $\alpha E_{0}^{2}=10^{-4}$. The arrows denote the first, $\mathrm{c} 1$, and the second, $\mathrm{c} 2$, critical points, where the curves change their slope from positive (forward-propagating modes) to negative (fast-light modes) and vice versa. In the present format, the light line in the nonlinear medium is pretty closed to the frequency axis and thus it is not depicted in these panels.

Therefore, all modes represented in panels (a)-(d) lay below the light line
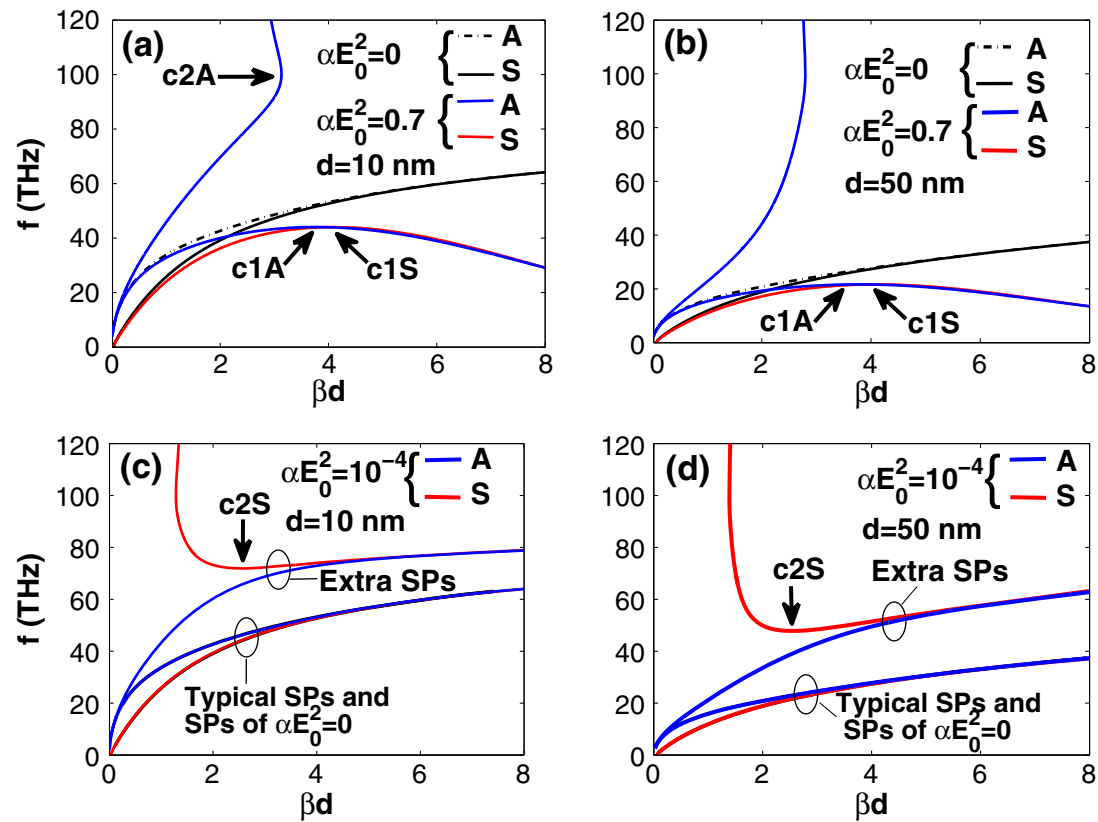

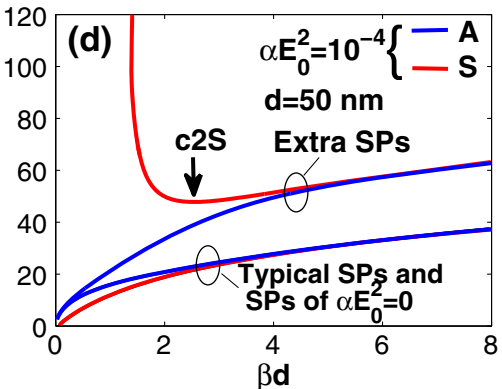

curves does not change with the inclusion of small losses, and $\operatorname{Re}\left(n_{e f f}\right)>0$ (i.e., $\left.v_{p h}>0\right)$ for the modes with negative slope of dispersion $\left(v_{g}<0\right)$ in Fig. 1a. As a result, these modes with positive total energy flux, $v_{e}>0, v_{p h}>0$ and $v_{g}<0$ are fast-light SPs.

In addition to the typical fast-light modes (modes with negative slope of dispersion in Fig. 2a which belong to the typical branches), extra antisymmetric SPs with negative slope of dispersion can also be supported for frequencies greater than the critical value $f_{c 2 A}=100.2 \mathrm{THz}\left(\beta_{c 1 S} d=\right.$ $3.131)$ shown by the arrow in this figure. Although these modes are less confined to the waveguide, as it is seen by comparing Fig. 3c with d, they can be excited at field intensities considerably smaller than those that are required to excite the typical fast-light modes. Figure 2 illustrates the antisymmetric nature of four SP modes shown in Fig. 2a at $f=30$ and $110 \mathrm{THz}$, and allows one to estimate their confinement and the relative strengths of the electric field
Fig. 3 Electric field

components $E_{x}$ and $E_{z}$ for four antisymmetric guided modes in Fig. 2a at $f=30$ and $110 \mathrm{THz}$. In accordance with the schematic of system illustrated in Fig. 1, the vertical black lines represent graphene layers. From panels (a) and (b), it is seen that forward modes can be supported for lower values of the electric field intensities, as compared with panels (c) and (d). From the comparison between panels (c) and (d), it is clear that extra fast-light SPs can be supported with considerably smaller values of the electric field intensities
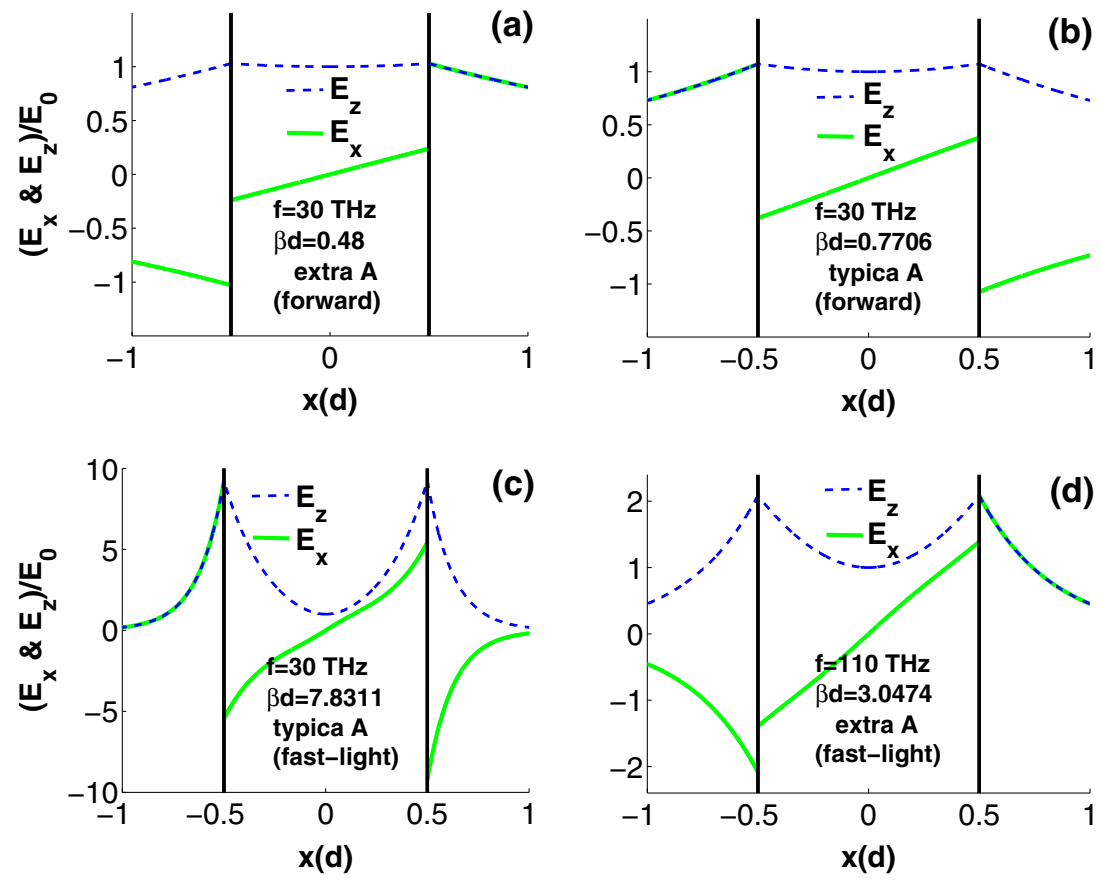
components. Since the electric field profiles of the symmetric modes are similar, they are not represented in the figure.

The considered examples show that the presence of a self-focusing Kerr medium at higher intensities $(0.7 / \alpha)$ profoundly alters the dispersion of SPs of a linear graphene PPW, giving birth to the typical fast-light guided plasmon modes and an extra branch of forward/fast-light SPs. Taking $\alpha E_{0}^{2}=0.7$ and increasing $d$ up to $50 \mathrm{~nm}$ causes the typical SPs to be supported at lower frequencies, as can be seen from Fig. 2b. There is also an additional branch of forward-propagating antisymmetric SPs in this case. Also noteworthy is that the typical antisymmetric and symmetric branches change their slopes at $f_{c 1}=21.66 \mathrm{THz}$ for normalized wave numbers exceeding $\beta_{c 1 S} d=3.647$ and $\beta_{c 1 S} d=4.042$, as indicated by arrows in Fig. $2 \mathrm{~b}$.

In case of applying light with lower intensity $\left(10^{-4} / \alpha\right)$, one can see from Fig. 2c that:

(i) GKG PPW supports four guided plasmon modes, two of which are typical and two are extra modes of antisymmetric and symmetric kinds.

(ii) The dispersion branches of the typical SP modes in this case are almost the same as those of the linear graphene PPW, with $\alpha E_{0}^{2}=0$

(iii) Dispersion curve of the additional antisymmetric SPs has positive slope similar to the linear modes, whereas the additional symmetric modes are fastlight SPs for $f>f_{c 2 S}=71.95 \mathrm{THz}$ and $\beta d<$ $\beta_{c 2 S} d=2.542$, as indicated by the arrow in Fig. 2c.
As it is also evidenced by Fig. 2d, an increase in the plate separation results in the SPs being supported at lower frequencies and with weaker localizations at the waveguide. The critical values of the normalized wave number and frequency are seen to be 2.542 and $47.79 \mathrm{THz}$. Hence, the spectral positions of the critical points can be notably shifted by varying the thickness of the waveguide or the intensity of the excitation field. Another point needs to be emphasized here is that as far as the thickness of the Kerr slab is taken appropriately (e.g., smaller than $1 \mu \mathrm{m}$, see refs. [14] and [15]) and appropriate values of the light intensity are applied, the forward and fast-light SPs can be supported by GKG PPW.

The effect of changes in the light intensity at the center of the waveguide on the dispersion of critical points are shown in Fig. $4 \mathrm{a}, \mathrm{b}$. Figure $4 \mathrm{a}$ shows that the changes in $\beta_{c 1} d$ are almost the same when $\alpha E_{0}^{2}$ is varied for $d=10$ and $50 \mathrm{~nm}$, and also that the mode degeneracy may occur at fixed frequencies for $0.14<\alpha E_{0}^{2}<0.7$; a similar behavior has also been reported for metallic waveguides [29]. A clear difference between the dispersion curves for the waveguides of different thicknesses is seen from Fig. $4 \mathrm{~b}: f_{c 1}$ shifts to lower values with an increase in the nonlinear part of the permittivity.

Since the most significant advantage of the graphenebased plasmonic structures over the similar metal-based ones is their tunability via the adjustment of the chemical potential, which can be achieved by gating or chemical doping, we illustrate this effect on the critical points of the SPs dispersion in Fig. 4c, d. Figure $4 c$ shows that the mode
Fig. 4 Effect of changes in the nonlinear part of the dielectric permittivity of the core medium on (a) normalized wave number and (b) frequency of the first critical points shown in Fig. 2a and b. Panels (c) and (d) are similar to panels (a) and (b) except that they illustrate the impact of changes in the chemical potential
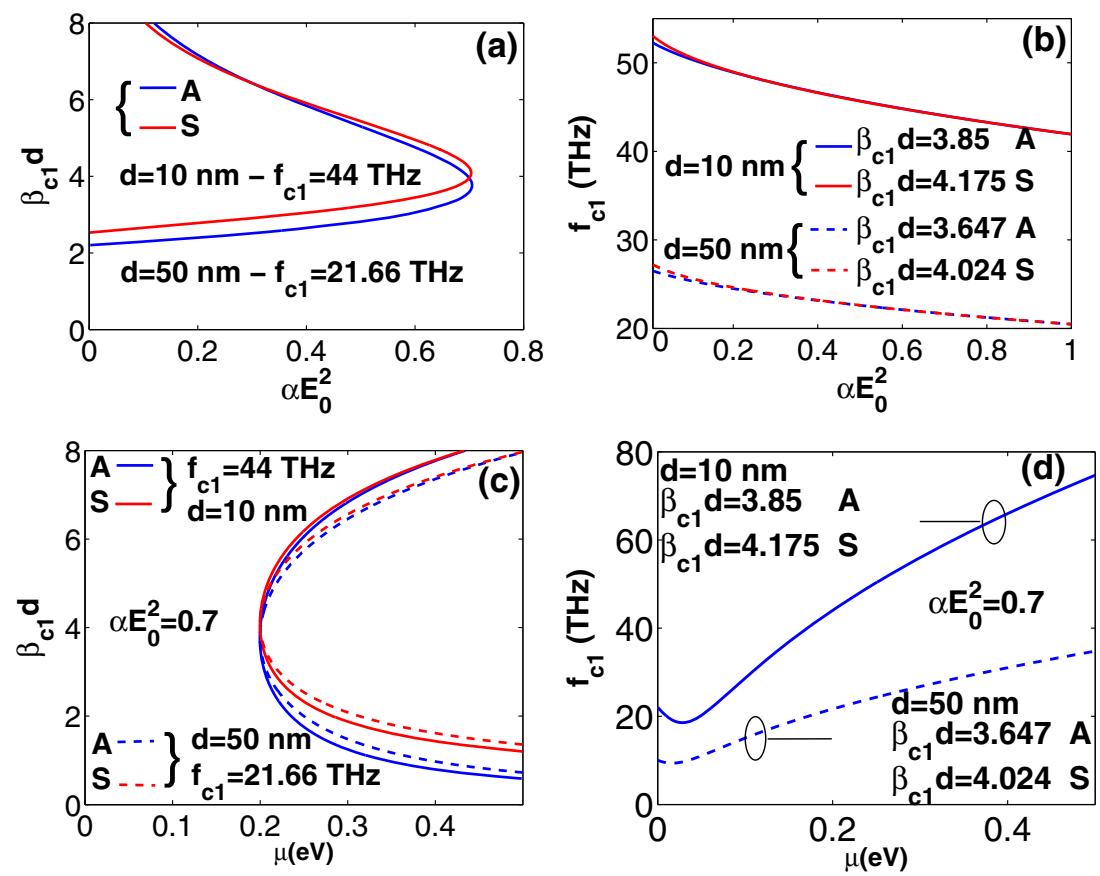
degeneracy can take place for different values of the chemical potential. Note that the dispersions of both symmetric and antisymmetric modes are almost the same in Fig. 4d. Besides, it is seen from this figure that there is an ascending or descending trend in frequency dispersion for different values of $\mu$. It is therefore clear that amending $\mu$ effectively adjusts the SPs characteristics. As a consequence, one can switch between the forward-propagating and fast-light SPs supported by the GKG PPW by tuning the graphene chemical potential or $\alpha E_{0}^{2}$. We have also investigated (results not shown) the aforementioned tunable characteristics for the second critical point, $c 2$. It has been seen that, as for the $c 1$ points, the frequency and wavenumber of the $c 2$ SPs as well as the slope-sign of their dispersion curves can be effectively adjusted by changing $\alpha E_{0}^{2}$ and $\mu$.

To get more insight into the characteristics of the fastlight SPs, the dielectric constant of the Kerr medium as a function of frequency (panels (a) and (b)) and the field intensity at the center of the waveguide (panel (c)) are represented in Fig. 5. Notice that panels (a) and (b) are presented for antisymmetric and symmetric SPs illustrated in panels (a) and (c) of Fig. 2, respectively. Figure 5c is also consistent with Fig. 4a. The similar trend can also be seen for the case we plot $\varepsilon_{1}$ versus $\alpha E_{0}^{2}$ for the symmetric modes of Fig. 2c.

Large and physically meaningless values of the upper branch of $\varepsilon_{1}$ with negative slope in panel (a) correspond to the typical antisymmetric fast-light SPs in Fig. 2a, while the lower branch with negative slope in this panel with smaller values of $\varepsilon_{1}$ corresponds to the extra fast-light-guided plasmon modes. This is the reason the modes with negative slope of the typical branches, which exist for large values of the wavenumber, can be supported for high intensities of the light. This point is also in agreement with the large values of the field components in Fig. 3c. Notice that the same thing holds for the case of Metal-Kerr-Metal problem investigated in refs. [24] and [25]. In contrast, the sensible values of the permittivity for the modes with negative slope in panel (b) are in accordance with the extra symmetric fast-light SPs in Fig. 2c with $\beta_{c 2 S} d<2.542$ for which a feasible value of the light intensity is considered at the center of the waveguide. From a practical point of view, considering $\alpha E_{0}^{2}=10^{-4}$ is more sensible than that of 0.7 and, we presented the results for both values of high and low intensities of light to have more extensive consideration of this problem.

Finally, we give a comparison between the GKG PPW and the similar systems studied recently [16, 27]. In the case where the nonlinear Kerr material surrounds the $G$ PPW (ref. [27]), the dispersion relation of our system can be found numerically without the knowledge of the exact field distribution in the nonlinear media. By having the field intensity at the interface of graphene and the nonlinear material, it would be possible to find the entire field distribution. In contrast, in this work, the core medium of the PPW is considered as a Kerr-type nonlinear material, and obtaining the exact dispersion relation of the system would not be possible without having the field distribution inside the core medium. Consequently, the process of solving the problem presented in this study is different than the previously reported one [27]. Moreover, the same as a typical G PPW, the structures analyzed in ref. [27] are capable of supporting only two branches of guided plasmonic modes with
Fig. 5 Panels (a) and (b) show the dielectric constant of the Kerr-type core medium versus frequency for the antisymmetric and symmetric branches of SPs shown in panel (a) and (c) of Fig. 2 , respectively. Panel (c) shows dependence of the dielectric constant on the intensity of the light at the center of the waveguide for the antisymmetric modes of Fig. 1a. The similar trend is also observed for the symmetric modes in accordance with panel (b)
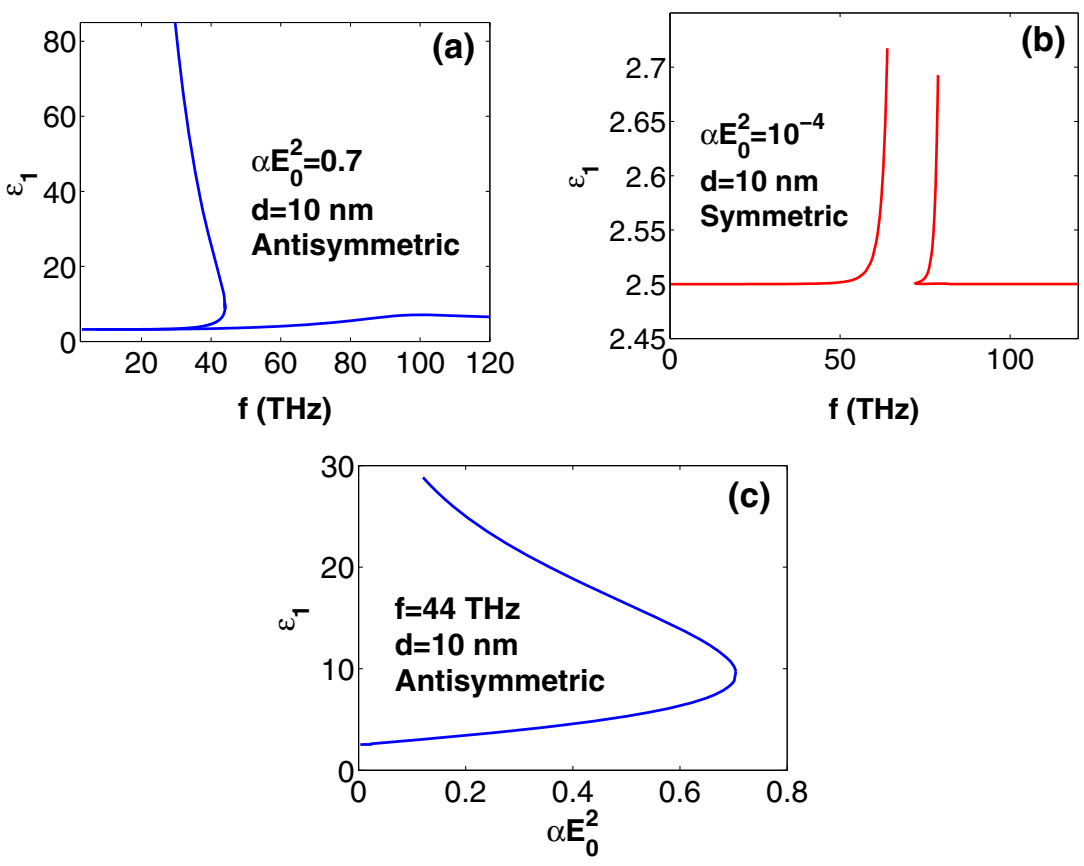
positive slope of dispersion. Another point should be highlighted here is that in ref. [16], considering the nonlinear optical response of graphene in the calculations, it has been shown that a G PPW with linear core medium and linear cladding media, supports three types of nonlinear modes; symmetric, antisymmetric, and asymmetric. In contrast, the modes supported by our system are either symmetric or antisymmetric. In addition to supporting extra branch(es) of SPs, there is a possibility of having modes with negative slope of dispersion in GKG PPW.

\section{Conclusions}

We have analytically studied the plasmon dispersion of a symmetric graphene/Kerr-medium/graphene parallel-plate waveguide. We found that, in addition to the typical guided forward-propagating modes of a double-layer of graphene with linear core medium, our structure supports additional branches of antisymmetric and symmetric SPs. We also showed that it is possible to excite fast-light SPs, modes with negative slope of dispersion and positive energy flux, in the waveguide. One can switch between the forward and fast-light SPs by changing the light intensity or the chemical potential of graphene.

Acknowledgments This work is supported by projects DPTHAMIT, ESF-EPIGRAT, and NATO-SET-181, and by TUBITAK under Projects Nos. 107A004, 109A015, 109E301, and 110T306. E.O. and H.C. acknowledge partial support from the Turkish Academy of Sciences. I.D.R. gratefully acknowledges the Ministry of Education and Science of the Russian Federation for its Grant 14.B25.31.0002.

\section{References}

1. Novoselov KS, Geim AK, Morozov SV, Jiang D, Zhang Y, Dubonos SV, Grigorieva IV, Firsov AA (2004) Electric field effect in atomically thin carbon films. Science 306:666

2. Bonaccorso F, Sun Z, Hasan T, Ferrari AC (2010) Graphene photonics and optoelectronics. Nat Photonics 4:611

3. Grigorenko AN, Polini M, Novoselov KS (2012) Graphene plasmonics. Nat Photonics 6:749

4. Rupasinghe C, Rukhlenko ID, Premaratne M (2014) Spaser made of graphene and carbon nanotubes. ACS Nano 8:24312438

5. Zhu W, Rukhlenko ID, Premaratne M (2013) Graphene metamaterial for optical reflection modulation. Appl Phys Lett 102: 241914

6. Zhu W, Rukhlenko ID, Premaratne M (2013) Graphene-enabled tunability of optical fishnet metamaterial. Appl Phys Lett 102:121911

7. Vakil A, Engheta N (2011) Transformation optics using graphene. Science 332:1291

8. Udagedara I, Rukhlenko ID, Premaratne M (2011) Complex- $\omega$ approach versus comple- $k$ approach in description of gain-assisted SPP propagation along linear chains of metallic nanospheres. Phys Rev B 83:115451
9. Udagedara I, Rukhlenko ID, Premaratne M (2011) Surface plasmon-polariton propagation in piecewise linear chains of composite nanospheres: the role of optical gain and chain layout. Opt Express 19:19973

10. Handapangoda D, Rukhlenko ID, Premaratne M (2013) Analytical study of optimal design and gain parameters of double-slot plasmonic waveguides. J Opt 15:035006

11. Handapangoda D, Rukhlenko ID, Premaratne M (2012) Optimizing the design of planar heterostructures for plasmonic waveguiding. J Opt Soc Am B 29:553-558

12. Handapangoda D, Premaratne M, Rukhlenko ID, Jagadish C (2011) Optimal design of composite nanowires for extended reach of surface plasmon-polaritons. Opt Express 19:16058

13. Handapangoda D, Rukhlenko ID, Premaratne M, Jagadish C (2010) Optimization of gain-assisted waveguiding in metaldielectric nanowires. Opt Lett 35:4190

14. Hanson GW (2008) Quasi-transverse electromagnetic modes supported by a graphene parallel-plate waveguide. J Appl Phys 104:084314

15. Hajian H, Soltani-Vala A, Kalafi M (2013) Optimizing terahertz surface plasmons of a monolayer graphene and a graphene parallel plate waveguide using one-dimensional photonic crystal. J Appl Phys 114:033102

16. Smirnova DA, Gorbach AV, Iorsh IV, Shadrivov IV, Kivshar YuS (2013) Nonlinear switching with a graphene coupler. Phys Rev B 88:045443

17. Liu M, Yin X, Zhang X (2012) Double-layer graphene optical modulator. Nano Lett 12:1482

18. Koppens FHL, Chang DE, Garcia FJ (2011) de Abajo, Graphene plasmonics a platform for strong light-matter interactions. Nano Lett 11:3370

19. Hendry E, Hale PJ, Moger J, Savchenko AK, Mikhailov SA (2010) Coherent nonlinear optical Response of graphene. Phys Rev Lett 105:097401

20. Nesterov ML, Bravo-Abad J, Nikitin A-Yu, Garcia-Vidal FJ, Martin-Moreno L (2013) Graphene supports the propagation of subwavelength optical solitons. Laser Photonics Rev 7: L7

21. Huang JH, Chang R, Leung PT, Tsai DP (2009) Nonlinear dispersion relation for surface plasmon at a metal-Kerr medium interface. Opt Commun 282:1412

22. Chen Q, Wang ZH (1993) Exact dispersion relations for TM waves guided by thin dielectric films bounded by nonlinear media. Opt Lett 18:260

23. Davoyan AR, Shadrivov IV, Kivshar Yu-S (2008) Nonlinear plasmonic slot waveguides. Opt Express 16:21209

24. Rukhlenko ID, Pannipitiya A, Premaratne M (2011) Dispersion relation for surface plasmon-polaritons in metal/nonlinear -dielectric/metal slot waveguides. Opt Lett 36:3374

25. Rukhlenko ID, Pannipitiya A, Premaratne M, Agrawal GP (2011) Exact dispersion relation for nonlinear plasmonic waveguides. Phys Rev B 84:113409

26. Wang L, Cai W, Zhang X, Xu J (2012) Surface plasmons at the interface between graphene and Kerr-type nonlinear media. Opt Lett 37:2730

27. Hajian H, Soltani-Vala A, Kalafi M, Leung PT (2014) Surface plasmons of a graphene parallel plate waveguide bounded by Kerr-type nonlinear media. J Appl Phys 115:083104

28. Falkovsky LA (2008) Optical properties of graphene. J Phys: Conf Series 129:012004

29. Davoyan A, Shadrivov IV, Bozhevolnyi SI, Kivshar YS (2010) Backward and forward modes guided by metal-dielectric-metal plasmonic waveguides. J Nanophoton 4:043509

30. Feigenbaum E, Kaminski N, Orenstein M (2009) Negative dispersion: a backwar d wave or fast light? Nanoplasmonic examples. Opt Exp 17:18934 